\title{
Use of Generalized Regression Neural Network in Authorship Attribution
}

\author{
R. Chandrasekaran \\ Department of Statistics \\ Madras Christian College \\ Tambram, Chennai, INDIA-600 059
}

\author{
G. Manimannan \\ Department of Statistics \\ Madras Christian College \\ Tambram, Chennai, INDIA-500059
}

\begin{abstract}
Identification of authorship of writings of unknown authorship is a special type of problems in the field of Stylometry. In this paper, the classification of articles of ambiguous authorship to the articles written by contemporary Tamil scholars of the same period, namely Mahakavi Bharathiar (MB), Subramaniya Iyer (SI), and T. V. Kalyanasundaram (TVK) is discussed. During the preindependence period, these three popular scholars had written number of articles on India's Freedom Movement in the magazine called, India. Initially, all the three writers contributed their articles by attributing their names. Later, all the three patriots wrote articles on the same theme for anonymous publications without mentioning their names due to the oppressive attitude of the then British regime.
\end{abstract}

Over the last two decades, the application of Artificial Neural Network models has increased considerably in areas of pattern classification and recognition problems in the field of Stylometry. In the present research, an attempt is made to apply the Generalised Regression Neural Network to the problem of authorship attribution for articles of ambiguous authorship and to assign them to the contemporary writers of the same period. Different sets of variables such as morphology and function words are made use of for classification purposes. Subsequently, results of authorship attribution are discussed.

\section{KEYWORDS}

Stylometry, Authorship attribution, Artificial Neural Network, Generalized Regression Neural Networks.

\section{INTRODUCTION}

Stylometry is the study of the quantifiable of human language, or the statistical analysis of literary style (Holmes, 1995; Holmes and Forsyth, 1995). This involves attempting to formally capture the creative, unconscious elements of language particular to individual writers and speakers. Although researchers have studied writing for centuries, the discipline of stylometry is fairly recent, and while its origins date back to the late $19^{\text {th }}$ century, the field as it is now began with work on the Federalist Papers in 1968 (Mosteller and Wallace, 1968). The problem of authorship attribution began with discipline known as Stylometry. Stylometry mainly concerns itself with authorship attribution studies, although chronological studies on the dating of work within the corpus of an author have also investigated. Writing in a forensic background, Bailey (1979) proposed three rules to define the situation necessary for authorship attribution:

1. The number of putative authors should constitute a well- defined set.
2. The lengths of the writings should be sufficient to reflect the linguistic behavior of the author of the disputed text and also those of the candidates.

3. The texts used for comparison should be commensurate with the disputed writing.

A computational stylistic study of doubtful authorship should involve comparisons of the disputed text with works by each of the possible candidate authors using suitable statistical tools on quantifiable features of the texts-features which reflect the style of the writing as defined above.

One modern addition to the tools available for the computational stylometry is that of the Artificial Neural Network (ANN). These are the computational methods closely based on the concept of biological neuron, the idea being that simple, trained processing elements will result in much more difficult behavior when used in combination.

In recent years, many scholars have successfully demonstrated that this technique of machine learning field can be applied to authorship attribution. Merriam and Mathews (1993, 1994) have trained a multi layer perception network to distinguish the works of Shakespeare and Marlowe. Tweedie et al. (1996) have provided a useful review of the applications of ANNs in the area of computational stylometry and have used this machine-learning package for the reanalysis of the Federalist Papers. Kjell (1994) have taken up authorship study using letter-pair frequency features with neural network classification. Recently, authorship identification problem is also attempted by the authors using the Radial Basis Function Network (Chandrasekaran and Manimannan, 2008). The present study attempts to use the Generalised Regression Neural Network (GRNN) as one of the suitable neural network classification tool.

\section{ARTIFICIAL NEURAL NETWORK}

An Artificial Neural Network (ANN) is a mathematical model represented by interlinked simple computational elements, called neurons that could compute, learn, remember and optimize the way a human brain works (Bishop, 2003; Heykin, 2001; Wasserman, 1989, 1993). The neurons in ANN are called nodes. The interconnected nodes (neurons) are arranged into several layers namely, the input, intermediate (hidden) and the output layers. Depending on the signals (data) transmitted by various nodes, a set of outputs is computed by the nodes that received the signals from the other nodes.

Initially the network should be subjected to learning process. The network must learn decision surfaces from a set of training patterns so that these training patterns are classified correctly (Gose, Johnsonbaugh and Jost, 1997). After training, the network must also be able to generalize, that is, correctly classify test patterns it has never seen before. Usually any 
neural network should be such that it has the ability to learn well and also to generalize well.

The general procedure is to have the network learn the appropriate weights from a representative set of training data. In all but the simplest cases, however, direct computation of the weights is difficult. Instead, learning starts off with random initial weights and adjusts them in successive iterations, until the required outputs are produced.

The supervised and the unsupervised learning methodologies are adapted by the ANN. In supervised learning, the objective is to predict one or more output variables from one or more input variables (Bishop, 2003; Ripley, 1996). In the unsupervised learning, there are no target variables. The network trains itself to extract the features from the input variables using which the input variable themselves can be predicted.

Tweedie et. al. (1996) discussed the application of neural networks in stylometry and their usefulness for a number of reasons:

1. Neural networks can learn from the data themselves. Implementing a rule-based system in linguistic computing may become complex as the number of distinguishing variables increases and even the most complex rules may still not be good enough to completely characterize the training data. In essence, neural networks are more adaptive.

2. Neural networks can generalize. This ability is particularly required in the literary field, as only limited data may be available.

3. Neural networks can capture non-linear interactions between input variables.

4. Neural networks are capable of fault tolerance. Hence a particular work, which is not in line with the usual writing style of an author, will not affect the network to a considerable extent. Thus neural networks appear to promise much for the field of stylometry. Their application would appear to be worthy of investigation.

The pioneering work in the application of neural nets in Stylometry was undertaken by Merriam and Mathews (1993). In their paper, a very small set of function word frequencies is used as input to a multiplayer perceptron (a neural net having a hidden layer) to examine four plays that have been attributed both to Shakespeare and John Fletcher.

In the present context an attempted is made to use the concepts of Radial Basis Function Network for the authorship attribution problem.

\section{GENERALIZED REGRESSION NEURAL NETWORK}

The Generalized Regression Neural Network is a kernel based feed forward neural network with an architecture having three layers namely, the input, hidden and output layers (Powell, 1992; Wasserman, 1989, 1993). The Generalized Regression Neural Networks use the kernel or Basis Function method which is introduced as a technique for performing exact nonlinear interpolation tasks on a set of data points in a multidimensional space (Bishop, 2003; Powell 1992, Wasserman 1993).
A number of basis functions such as Gaussian, quadric, multiquadric, cubic and thin-plate-spine, have been proposed by many authors. A direct implementation of this method leads to the feed forward neural network with an architecture having three layers namely, the input, hidden and output layers. The input layer corresponds to $\mathbf{x}_{\mathrm{i}}$, the hidden layer to basis function $\varphi($.$) 's and the output layer to f\left(\mathbf{x}_{\mathrm{i}}\right)$ or $\mathbf{y}_{\mathrm{i}}$. The net of each of the hidden layer is non-linear while that of output is linear. Usually, each of the basis function $\varphi($.) associated with the hidden node $\boldsymbol{i}$ is localized yielding greater value when the input $\mathbf{x}$ is closer to its center $\mathbf{x}_{\mathrm{i}}$ and tends to zero as $\mathbf{x}$ deviates from the center. In general, the nonlinear function $\varphi$ transforms a nonlinearly separable problem into a linearly separable one. The exact interpolation needs $\mathbf{n}$ hidden nodes for $\mathbf{n}$ input data points and hence grows with the number of data points. Instead of having a hidden node for each of the data point, a reduced number of hidden nodes could be considered. This could be achieved by selecting a fixed number $\boldsymbol{C}$ of data points chosen at random from the input data set, using orthogonal least square or K-means clustering algorithm.

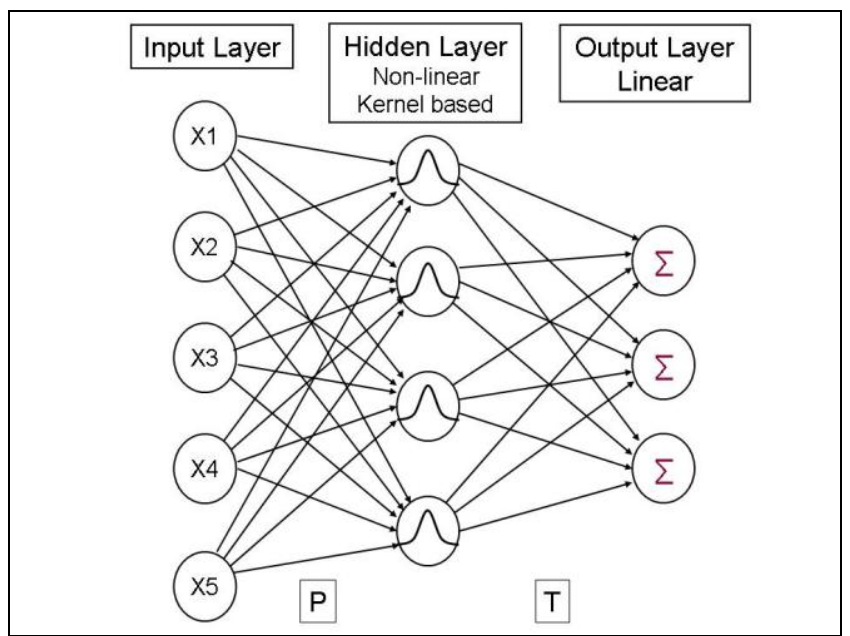

Figure 1. Generalized Regression Neural Network $\mathrm{y}=f(\mathrm{x})$.

\section{DATABASE}

The present study deals with the literary works of three contemporary Tamil scholars, namely, Mahakavi Bharathi (MB), T. V.Kalyanasundaranar (TVK), and Subramaniya Iyer (SI). In the Pre-Independence period, these three scholars have written number of articles on India's Freedom Movement in the magazine called India. Initially, all the three scholars have written articles by attributing their names. The oppressive attitude of the then British Regime made all the three writers to write articles on the same topic anonymously in the same magazine. For this quantitative attribution study, all attributed articles of these three scholars written on India's Freedom Movement in the year 1906 are considered.

Our study is based on nineteen articles of MB, seven of TVK and six of SI and twenty-three un-attributed articles. Twenty four function words and eighteen morphological variables have been used to quantify each sentence. The exact lists of variables of this study with their meanings are given in Table 1 and Table 2 . 
Table 1. List of Twenty Four Function Words

\begin{tabular}{|l|l|l|l|}
\hline $\begin{array}{c}\text { Function } \\
\text { Words }\end{array}$ & Translation & $\begin{array}{l}\text { Function } \\
\text { Words }\end{array}$ & Translation \\
\hline Um & Also & Ai & Unmarked \\
Entraal & For & Lodu & With \\
Aavatu1 & For & Aall & With \\
Aaka & As & Ukku & To \\
Mikavum & Very much & Atu & My \\
Pola & Like & Ill & In \\
Entru & For & Utan & With \\
Pearil & On & Um1 & At every \\
Irrunthu & From & Enum & At least \\
Kooda & Also & Utaiya & Of \\
U1 & Inside & Pattri & About \\
\hline
\end{tabular}

Table 2. Lists of Morphological Variables of this Study with Abbreviations

\begin{tabular}{|l|l|}
\hline \multicolumn{1}{|c|}{ Abbreviations } & \multicolumn{1}{|c|}{ Variables Name } \\
\hline P_NOUN & Nouns \\
P_INT & Introductory \\
P_INF & Intensifiers \\
P_PRO & Pronouns \\
P_NUME & Numerals \\
P_TWO & Two letter Words \\
P_THRE & Three letter Words \\
P_FOUR & Four letter Words \\
P_VOWE & Vowels \\
P_VERB & Verbs \\
P_SYLLA & Syllables \\
P_POST & Postpositions \\
P_CLITIC & Clitics \\
P_CASE & Case Markers \\
P_ADVERB & Adverbs \\
P_CONJUN & Conjunctions \\
TENSES & Tenses \\
VOICES & Voices \\
\hline
\end{tabular}

A chi-square analysis of the nineteen articles of $\mathrm{MB}$ establishes that these articles do not differ from one another in terms of the frequency distribution of occurrence of these stylistic features. Similar results were obtained in the case of other two scholars (Manimannan and Bagavandas, 2001). Thus, each article is converted as a raw data matrix and these raw data matrices form the basis for this data description. Hence the nineteen articles of Mahakavi Bharathiar (MB) consist of three hundred and fifty-three sentences, the seven articles of Subramaniya Iyer (SI) consist of three hundred and fifteen sentences and six articles of T. V. Kalyanasundaram (TVK) consist of three hundred and eighty-two sentences. The data matrix of $\mathrm{MB}$ is of size $(353 * 18)$, of SI is of size $(315 * 18)$ and of TVK is of size $(382 * 18)$ in the case of morphological variables and they are $(353 * 24),(315 * 24)$ and $(382 * 24)$ respectively in the case of function words. The entire twenty-three un-attributed articles consist of three hundred and seventy-five sentences. Recently, Chandrasekaran and Manimannan (2012) have used the Radial Basis Function Network to identify writers of the articles of ambiguous authorship considered in this paper.

\section{RESULTS AND DISCUSSION}

Nineteen articles written by Mahakavi Bharathiar (MB), seven articles by Subramaniya Iyer (SI) and six articles by $\mathrm{T}$. V. Kalyanasundaram (TVK) have been considered for the analysis represented by the averages of the sampled sentences. The 32 data points representing the means of the corresponding sample writings of three different authors are used. The Data matrix (P) consists of 18 morphological variables, normalized, computed from the chosen articles. The Target matrix (T) has three rows, one row for each author, each row consisting of $0,1,2$ or 3 . This matrix element $t_{i j}$ is define as

$$
\begin{aligned}
& \mathrm{t}_{\mathrm{ij}}=\mathbf{i} \quad \text { if the sample } \mathbf{j} \text { in the data matrix } \mathbf{P} \\
& \text { corresponds to the author } \mathbf{i} \text {; }
\end{aligned}
$$

\section{0 otherwise.}

The generalized regression neural network is created with appropriate parameters and the data matrices. The articles of ambiguous authorship is also presented in the form of a Test matrix (X) consisting of 18 morphological variables, normalized, in the form of column vectors, each column corresponding to one sample article of unknown authorship. The entire analysis was also performed on the 24 normalised functional variables.

The Generalized Regression Neural Network (GRNN) available with the Neural Network Toolbox in the MATLAB software is used for the analysis. The GRNN routine creates as many neurons as there are input vectors in $\mathrm{P}$ in the hidden layer. Each neuron's weighted input is the distance between the input vector and its weight vector. The hidden layer weights are set to P-transpose. Each hidden neuron's net input is the element-by-element product of its weighted input with its bias. The hidden neuron's output is its net input passed through the radial basis function $R B F(\boldsymbol{m})=\exp \left(-\boldsymbol{m}^{2}\right)$, where $\boldsymbol{m}=$ net input $=\{\|$ weight vector - input vector $\|$ bias $\}$. Every bias in the hidden layer is set to $0.8326 /$ SPREAD, which gives radial basis function that crosses 0.5 at the weighted inputs of +/- SPREAD, where SPREAD lies between 0 and 1 . The larger the SPREAD smoother will be the approximation. SPREAD has been taken to be 0.9. The second layer's weight is set to $\mathrm{T}$ and has neurons with linear transfer function. The outputs of the second layer are calculated as the dot product of weights $(\mathrm{T})$ and the output vector of the hidden layer normalized by the sum of the output vector elements. The second layer does not have bias.

The program yielded the output which assigned all the 23 writings of unknown authorship to Mahakavi Bharathiar (MB) for morphological as well as functional variables. This result supported the claims made by many scholars that these 23 articles could have been written by Mahakavi Bharathiar (MB). 


\section{CONCLUSION}

Assignment of articles of ambiguous authorship to the contemporary Tamil scholars, namely Mahakavi Bharathiar (MB), Subramaniya Iyer (SI), and T. V. Kalyanasundaram (TVK), all of them belonging to of the same period, is taken up in the present research. Initially, all the three writers contributed their articles by specifying their names. The oppressive attitude of the then British regime compelled all the three patriots to write articles on the same theme for anonymous publications without mentioning their names.

During the last two decades, the application of Neural Network models has increased considerably in areas of pattern recognition and classification problems in the field of Stylometry. The authorship attribution problem is attempted using a Generalized Regression Neural Network for attributing the 23 articles of ambiguous authorship to one of the contemporary writers of the same period, first using morphological variables and then functional variables. All the articles are attributed to Mahakavi Bharathiar (MB). This result supported the claims made by many scholars that these 23 articles could have been written by Mahakavi Bharathiar (MB). Our recent research also supported the claim that these articles with ambiguous authorship were written by Mahakavi Bharathiar (MB).

\section{REFERENCES}

[1] Chandrasekaran, R. and Manimannan, G. (2008), Neural Network Classification and Authorship Attribution of Articles of Unknown Authorship Using Radial Basis Function, International Journal of Engineering Research and Technology (IJERT) ISSN: 2278- 0181, 2012

[2] Bailey, R. W. (1979), The Future of Computational Stylistics, Association for Literary and Linguistic Computing Bulletin, Vol. 7, 4-11. England.

[3] Bishop, C. M (2003), Neural Networks for Pattern Recognition (First Indian Edition), Oxford University Press, New Delhi

[4] Gose, E., Johnsonbaugh, R, and Jost, S (1997), Pattern Recognition and Image Analysis, Prentice Hall Inc., New Jersey.

[5] Heykin, S. (2001), Neural Networks : A Comprehensive Foundation (Second Edition), Pearson Education (Singapore), New Delhi.
[6] Holmes, D. I. (1995), The Analysis of Literary Style : A Review, Journal of Royal Statistical Society, Series A, Vol. 148, 328-334, England.

[7] Holmes D.I. and Forsyth, R.S. (1995), The Federalist Revisited: New Directions in Authorship Attribution, Literary and Linguistic Computing, 10, 111-127, England.

[8] Kjell, B. (1994), Authorship Determination Using Letterpair Frequency Features with Neural Network Classifiers, Literary and Linguistic Computing, Vol.9, 119-124, England.

[9] Manimannan, G. and Bagavandas, M. (2001), Authorship Attribution : The case of Bharathiar, National Conference on Mathematical and Applied Statistics, Department of Statistics, Nagpur University, Nagpur.

[10] Merriam, T. and Mathews, R. (1993), Neural Computation in Stylometry I: An Application to the Works of Shakespeare and Fletcher, Literary and Linguistic Computing, Vol.8, 203-209, England.

[11] Merriam, T. and Mathews, R. (1994), Neural Computation in Stylometry II: An Application to the Works of Shakespeare and Marlowe, Literary and Linguistic Computing, Vol.9, 1-6, England.

[12] Mosteller, F. and Wallace, D. L. (1968), Inference and Disputed Authorship : The Federalist Papers, AddisionWesley, Messachusetts.

[13] Powell, M. J. D. (1992), The Theory of Radial Basis Functions Approximation, in Advances of Numerical Analysis , pp. 105-210, Clarendon Press, Oxford.

[14] Ripley, B.D. (1996), Pattern Recognition and Neural Networks, Cambridge University Press.

[15] Tweedie, F. J, Singh, S., and Holmes, D. I. (1996), Neural Network Applications in Stylometry. The Federalist Papers, Computers and the Humanities, 39(1), 1-10, 1996.

[16] Wasserman, P.D (1989), Neural Computing : Theory and Practice, Von Nostrand Reinhold, New York

[17] Wasserman, P.D (1993), Advanced Methods in Neural Computing, Von Nostrand Reinhold, New York. 